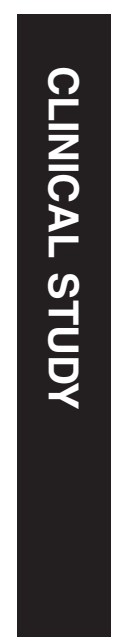

\section{Safety and efficacy of simultaneous bilateral 25-gauge lens-sparing vitrectomy for vascularly active stage 4 retinopathy of prematurity}

PK Shah, V Narendran and N Kalpana

\begin{abstract}
Purpose To report the safety and efficacy of simultaneous bilateral 25-gauge lens-sparing vitrectomy for vascularly active stage 4 retinopathy of prematurity (ROP).

Methods Retrospective, noncomparative, consecutive case series. Twenty eyes of 10 babies who presented with vascularly active stage 4 ROP in both the eyes underwent simultaneous bilateral 25-gauge lens-sparing vitrectomy. After completing surgery for one eye, the other eye was re-prepped as performed before starting any new case of a different patient and an entire new set of disposable 25-gauge instruments were used. During the post-operative period parents were advised to keep separate eye drops for each eye and to wash their hands in between switching the eyes to put the drops.

Results The mean follow-up was 8.7 months (range 4-17 months). None of the cases developed any signs of infection. The anatomic success rate for stage 4 a was $100 \%$ (11/11 eyes) and for stage $4 \mathrm{~b}$ was $8 / 9$ eyes ( $89 \%$ ).

Conclusion These results show that simultaneous bilateral 25-gauge lens-sparing vitrectomy for stage 4 ROP is a safe and effective procedure with a good outcome provided both eyes of the baby are treated as eyes of two different patients.

Eye (2015) 29, 1046-1050; doi:10.1038/eye.2015.78; published online 22 May 2015
\end{abstract}

\section{Introduction}

Severe stage 3 retinopathy of prematurity (ROP) with plus disease usually presents by 35-37 weeks of post-conceptional age (PCA). ${ }^{1}$ Inadequate laser or no treatment at this stage may trigger the disease to progress to stage 4 and subsequently to stage 5 within 3-4 weeks. The timing of surgery for stage $4 \mathrm{ROP}$ is an important consideration just as stage 3 requires rapid laser when threshold or high-risk prethreshold is reached. The ideal timing for vitrectomy is when the vascular activity is reducing with detachment just beginning. This usually occurs around 40 weeks PCA, which is the due date of the child. ${ }^{2}$

Few cases present in this window period with bilateral vascularly active stage 4 ROP.

Vascularly active is defined as the presence of plus disease with active new vessels. Giving a gap of 2-3 weeks between vitrectomy for both eyes, to prevent devastating complications like bilateral endophthalmitis, ${ }^{3}$ could be disastrous for the second eye as the progression of retinal detachment in these cases is very fast and can quickly end in stage $5 \mathrm{ROP}$ and blindness. Also giving general anaesthesia again within a short duration could be risky in these preterm children. We present our experience in managing acute bilateral stage 4 ROP by doing simultaneous bilateral 25-gauge lens-sparing vitrectomy in the same sitting.

\section{Materials and methods}

This is a retrospective case series of babies who underwent bilateral simultaneous 25-gauge vitrectomy from November 2012 till February 2014. All the babies included in this study presented with bilateral acute stage 4 ROP with plus disease. All applicable institutional regulations concerning the ethical use of human volunteers were followed during this research and approval from the ethics committee was 
also taken. Detailed informed consent was taken from the parents explaining to them not only about the surgical complications like vitreous haemorrhage, iatrogenic retinotomies, need for lensectomy but also an increased risk of bilateral endophthalmitis. All surgeries were performed by a single surgeon (PKS). Under general anaesthesia after doing an indirect ophthalmoscopy standard three-port trans conjuctival 25-gauge vitrectomy was performed in one eye. Intravitreal triamcinolone was used in some for a more complete vitrectomy. ${ }^{4}$ At the end of the surgery partial fluid-air exchange was performed and sutures were applied only if sclerotomies were not adequately sealed. After completing one eye surgery, all the surgical drapes were removed and the other eye was re-prepped with povidone-iodide, re-draped using fresh set of sterile drapes and also the entire surgical staff rescrubbed, as performed for any new surgery of a different patient. A complete different set of sterile surgical instruments were used including new 25-gauge trocar and cannulas and the entire 25-gauge vitrectomy set. The surgical procedure was performed in the same way as the first eye and subconjunctival antibiotic and steroids injection was given at the end of each surgery. During the post-operative period, parents were advised to keep separate eye drops for each eye and to wash their hands in between putting the drops in each eye. All the babies were examined on day 1 after surgery, then 2 weeks and monthly thereafter.

\section{Results}

Twenty eyes of 10 babies were operated during the study period. The mean gestational age was 29.8 weeks (range 28-32 weeks) and mean birth weight was $1265 \mathrm{~g}$ (range 890-1700 g). The mean post-conceptional age at the time of surgery was 38.5 weeks (range 37-42 weeks) and the mean post natal age was 8.6 weeks (range 5-13 weeks). All cases had bilateral stage 4 ROP with plus disease.

Eighteen eyes were in zone 1 and two in zone 2. Eleven eyes had stage $4 \mathrm{a}$ while nine had $4 \mathrm{~b}$ ROP. Six eyes of the three cases were give prior laser before being referred to our hospital. Four eyes of two cases were given intravitreal avastin 1 week before vitrectomy to reduce the vascularity and all these eyes remained stable at final follow-up without giving any laser (Figure 1). Case 9 was referred to us after giving laser and avastin elsewhere. In five eyes of three patients avastin was given at the end of vitrectomy, as they had very vascularly active disease. Of these five eyes, endolaser was given in both eyes of one case, whereas delayed laser was given in one eye of the other patient when neovascularization returned 3 months after surgery. ROP regressed in the remaining two eyes with only avastin and vitrectomy without laser. The left eye of case 10, both pre- and intraoperative avastin was given (Table 1). Three eyes of the two cases developed glaucoma post-operatively, which was controlled by medications. Intravitreal triamcinolone to stain the vitreous during vitrectomy was used only in one of these

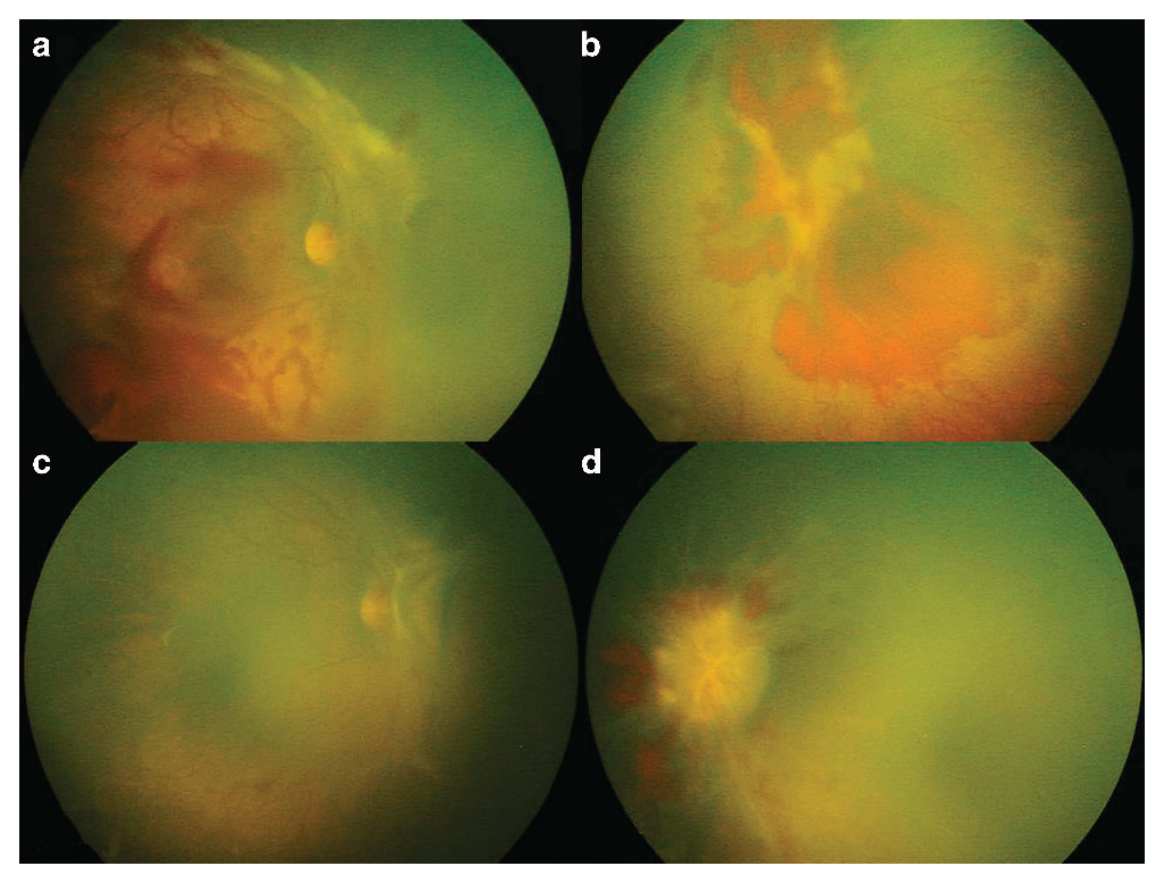

Figure 1 ( $a$ and b) Preoperative fundus pictures of right and left eyes showing stage $4 \mathrm{a}$ and $4 \mathrm{~b}$ respectively with vitreous haemorrhage. (c and d) Post-operative fundus pictures showing stable 4a and 4b ROP in right and left eyes respectively. Both eyes were given preoperative avastin and both regressed post vitrectomy without any laser. 
eyes. Lensectomy was performed in one eye with stage $4 \mathrm{~b}$, secondary to lens touch as the fibrous proliferation was very anterior. The mean follow-up was 8.7 months (range 4-17 months). None of the cases developed any signs of infection. All the nine cases of stage 4a remained stable till last follow-up (Figure 2). The anatomic success rate for stage $4 \mathrm{a}$ was $100 \%$. Only one eye with stage $4 \mathrm{~b}$ progressed to stage 5 . No prior or intraoperative laser or avastin was given in this eye. The anatomic success of stage $4 \mathrm{~b}$ was $8 / 9$ eyes (89\%). Nine eyes (45\%) developed high myopia ( $>6$-dioptre sphere). All these had finished at least a 6 month follow-up. In other cases, the refractive

Table 1 Baseline characteristics and treatment outcome

\begin{tabular}{|c|c|c|c|c|c|c|c|c|c|c|c|}
\hline Case no. & $\begin{array}{c}G A \\
\text { (weeks) }\end{array}$ & $B W(g)$ & $\begin{array}{c}P C A \\
\text { (weeks) }\end{array}$ & Eye & Zone & Stage & $\begin{array}{c}\text { Preoperative } \\
\text { laser }\end{array}$ & $\begin{array}{c}\text { Intraoperative } \\
\text { laser }\end{array}$ & $\begin{array}{c}\text { Preoperative } \\
\text { avastin }\end{array}$ & $\begin{array}{c}\text { Intraoperative } \\
\text { avastin }\end{array}$ & $\begin{array}{l}\text { Final } \\
\text { outcome }\end{array}$ \\
\hline \multirow[t]{2}{*}{1} & 32 & 1550 & 38 & RE & 1 & $4 a$ & $\mathrm{~N}$ & $\mathrm{~N}$ & Y & $\mathrm{N}$ & $\mathrm{F}$ \\
\hline & & & & LE & 1 & $4 b$ & $\mathrm{~N}$ & $\mathrm{~N}$ & Y & $\mathrm{N}$ & $\mathrm{F}$ \\
\hline \multirow[t]{2}{*}{2} & 29 & 890 & 37 & RE & 1 & $4 a$ & $\mathrm{~N}$ & $\mathrm{~N}^{\mathrm{a}}$ & $\mathrm{N}$ & $\mathrm{Y}$ & F \\
\hline & & & & LE & 1 & $4 a$ & $\mathrm{~N}$ & $\mathrm{~N}$ & $\mathrm{~N}$ & $\mathrm{Y}$ & $\mathrm{F}$ \\
\hline \multirow[t]{2}{*}{3} & 32 & 1700 & 40 & $\mathrm{RE}$ & 1 & $4 a$ & $\mathrm{~N}$ & Y & $\mathrm{N}$ & Y & $\mathrm{F}$ \\
\hline & & & & LE & 1 & $4 \mathrm{~b}$ & $\mathrm{~N}$ & Y & $\mathrm{N}$ & Y & $\mathrm{F}$ \\
\hline \multirow[t]{2}{*}{4} & 28 & 1000 & 39 & RE & 1 & $4 a$ & $\mathrm{Y}$ & $\mathrm{N}$ & $\mathrm{N}$ & $\mathrm{N}$ & F \\
\hline & & & & $\mathrm{LE}$ & 1 & $4 b$ & $\mathrm{Y}$ & $\mathrm{N}$ & $\mathrm{N}$ & $\mathrm{N}$ & F \\
\hline \multirow[t]{2}{*}{5} & 30 & 1500 & 38.4 & RE & 1 & $4 b$ & $\mathrm{Y}$ & $\mathrm{N}$ & $\mathrm{N}$ & $\mathrm{N}$ & F \\
\hline & & & & LE & 1 & $4 b$ & $\mathrm{Y}$ & $\mathrm{N}$ & $\mathrm{N}$ & $\mathrm{N}$ & F \\
\hline \multirow[t]{2}{*}{6} & 28 & 900 & 38 & RE & 1 & $4 a$ & $\mathrm{Y}$ & $\mathrm{N}$ & $\mathrm{N}$ & $\mathrm{N}$ & $\mathrm{F}$ \\
\hline & & & & $\mathrm{LE}$ & 1 & $4 a$ & $\mathrm{Y}$ & $\mathrm{N}$ & $\mathrm{N}$ & $\mathrm{N}$ & F \\
\hline \multirow[t]{2}{*}{7} & 29 & 940 & 42 & RE & 2 & $4 a$ & $\mathrm{Y}$ & $\mathrm{Y}$ & $\mathrm{N}$ & $\mathrm{N}$ & F \\
\hline & & & & $\mathrm{LE}$ & 2 & $4 a$ & Y & $Y$ & $\mathrm{~N}$ & $\mathrm{~N}$ & $\mathrm{~F}$ \\
\hline \multirow[t]{2}{*}{8} & 32 & 1450 & 39 & RE & 1 & $4 b$ & $\mathrm{~N}$ & $\mathrm{~N}$ & $\mathrm{~N}$ & $\mathrm{~N}$ & $\mathrm{U}$ \\
\hline & & & & $\mathrm{LE}$ & 1 & $4 b$ & $\mathrm{~N}$ & $\mathrm{~N}$ & $\mathrm{~N}$ & $\mathrm{~N}$ & F \\
\hline \multirow[t]{2}{*}{9} & 28 & 1420 & 38 & RE & 1 & $4 b$ & $\mathrm{Y}$ & $\mathrm{N}$ & $\mathrm{Y}$ & $\mathrm{N}$ & F \\
\hline & & & & $\mathrm{LE}$ & 1 & $4 \mathrm{~b}$ & $\mathrm{Y}$ & $\mathrm{N}$ & Y & $\mathrm{N}$ & $\mathrm{F}$ \\
\hline \multirow[t]{2}{*}{10} & 30 & 1300 & 38 & RE & 1 & $4 a$ & $\mathrm{~N}$ & $\mathrm{~N}$ & $\mathrm{Y}$ & $\mathrm{N}$ & $\mathrm{F}$ \\
\hline & & & & LE & 1 & $4 a$ & $\mathrm{~N}$ & $\mathrm{~N}$ & Y & Y & F \\
\hline
\end{tabular}

Abbreviations: BW, birth weight; F, favourable; GA, gestational age; LE, left eye; N, no; no., number; PCA, Post-conceptional Age; RE, right eye; $\mathrm{U}$, unfavourable; $\mathrm{Y}$, yes. ${ }^{\mathrm{a}}$ Delayed post-operative laser given 3 months after surgery.

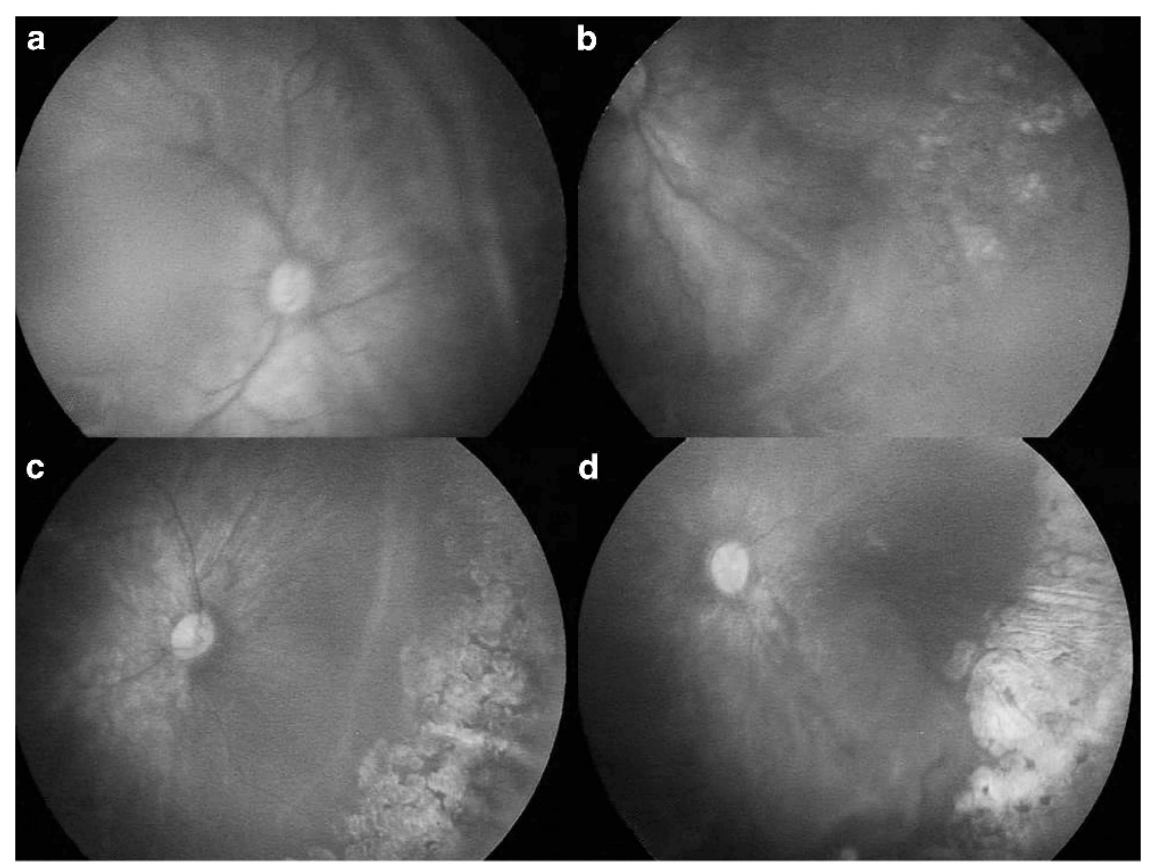

Figure 2 ( $a$ and b) Preoperative fundus picture of right and left eyes showing stage 4a ROP. (c and d) Post-operative fundus picture of both the eyes showing regressed $4 a$ ROP. 
error could not be assessed due to shorter follow-up in these infants. Three eyes had additional glaucoma. The corneal diameters for these three eyes were $12 \mathrm{~mm}$ for two and $11 \mathrm{~mm}$ for one eye.

\section{Discussion}

Bilateral simultaneous cataract surgeries have been reported in paediatric age group ${ }^{5,6}$ and in adults. ${ }^{7}$ However, it still remains quite controversial ${ }^{3,8}$ as one of the main reasons is risk of bilateral endophthalmitis, which could lead to bilateral visual loss. The reported incidence of endophthalmitis following simultaneous bilateral cataract surgery is between 0.15 and $1.1 \%$, as compared with $0.1 \%$ or less following unilateral or sequential cataract extractions. ${ }^{9}$ Endophthalmitis rates after adult 20- or 25-gauge vitrectomies is $0.03 \% .{ }^{10}$ Rate of endophthalmitis after vitrectomy for ROP is not known. ${ }^{11}$ We chose to perform bilateral simultaneous vitrectomy as delay of 2-3 weeks between the two eyes would have led to stage $5 \mathrm{ROP}$ and blindness in the latter eye. Moreover giving general anaesthesia twice within a short duration in these preterm babies can also be risky. None of our cases developed any signs of infection or endophthalmitis. This could be because we re-prepped the second eye in the same way as performed for any new surgical case of a different patient, and new set of disposable instruments were used and the entire surgical staff were also rescrubbed. Post-operatively the parents were instructed to use a different set of eye drops for each eye and to wash their hands in between applying the drops in each eye. The incidence of glaucoma in our study was was $15 \%(3 / 20)$, which is similar to the study by Iwahashi-Shima et al. ${ }^{12}$

No previous reports have focused specifically on bilateral simultaneous lens-sparing vitrectomy for acute stage 4a ROP. Azuma et al ${ }^{13}$ have reported bilateral simultaneous ROP-related vitrectomy but in their series of 22 eyes they did not mention how many of their cases underwent bilateral simultaneous surgery. Moreover their outcome was very poor (100\% failure) in cases with lens-sparing vitrectomy compared with the lensectomy vitrectomy group. In our study only one case required lensectomy because of lens touch. No pre- or intraoperative laser or avastin was given in this case. It is known that preoperative laser or avastin improves the final outcome. ${ }^{14,15}$ The anatomical outcome for stage $4 \mathrm{a}$ was $100 \%$ (11/11 eyes) and for $4 \mathrm{~b}$ was $89 \%$ ( $8 / 9$ eyes), which is similar to the studies published previously. ${ }^{16-18}$ Treatment option other than vitrectomy is scleral buckle, which has certain limitations. First, it is suitable for only anterior traction and not for posterior detachments. Second, it does not restore normal retinal anatomy and finally it causes anisometropia, which can lead to amblyopia. ${ }^{19}$ Observation is also an option in certain stage 4a detachments as some of these get better spontaneously. But these should not be progressive with the absence of plus disease. All our cases were active with plus disease.

Drawbacks of our study are its retrospective nature and a small sample size. Thus bilateral simultaneous vitrectomy for stage 4 ROP can be performed in cases where there is a simultaneous risk of fast progression to stage 5 ROP in both eyes. However all sterile precautions need to be followed for the second eye surgery as performed for any new case and parents also need to be counselled regarding the rare complication of bilateral endophthalmitis. We recommend further studies with larger numbers to support our findings.

\section{Summary}

\section{What was known before}

- Bilateral simultaneous intraocular surgeries are not routinely performed because of increased risk of bilateral endophthalmitis.

What this study adds

- Bilateral simultaneous vitrectomy for bilateral vascularly active stage 4 ROP can be performed.

\section{Conflict of interest}

The authors declare no conflict of interest.

\section{References}

1 Early Treatment For Retinopathy Of Prematurity Cooperative Group. Revised indications for the treatment of retinopathy of prematurity: results of the early treatment for retinopathy of prematurity randomized trial. Arch Ophthalmol 2003; 121: 1684-1694.

2 Shah PK, Narendran V, Kalpana N, Tawansy KA. Anatomical and visual outcome of stages 4 and 5 retinopathy of prematurity. Eye (Lond) 2009; 23: 176-180.

3 Henderson BA, Schneider J. Same-day cataract surgery should not be the standard of care for patients with bilateral visually significant cataract. Surv Ophthalmol 2012; 57: 580-583.

4 Shah PK, Narendran V, Kalpana N. Triamcinolone acetonide-assisted vitrectomy for stage 4 retinopathy of prematurity. Int Ophthalmol 2011; 31: 237-238.

5 Wagner RS. Simultaneous bilateral cataract surgery in children. J Pediatr Ophthalmol Strabismus 2012; 49: 138.

6 Magli A, Forte R, Rombetto L. Long-term outcome of primary versus secondary intraocular lens implantation after simultaneous removal of bilateral congenital cataract. Graefes Arch Clin Exp Ophthalmol 2013; 251: 309-314.

7 Sarikkola AU, Uusitalo RJ, Hellstedt T, Ess SL, Leivo T, Kivelä T. Simultaneous bilateral versus sequential bilateral cataract surgery: Helsinki Simultaneous Bilateral Cataract 
Surgery Study Report 1. J Cataract Refract Surg 2011; 37: 992-1002.

8 Arshinoff SA. Same-day cataract surgery should be the standard of care for patients with bilateral visually significant cataract. Surv Ophthalmol 2012; 57: 574-579.

9 Ramsay AL, Diaper CJ, Saba SN, Beirouty ZA, Fawzi HH. Simultaneous bilateral cataract extraction. J Cataract Refract Surg 1999; 25: 753-762.

10 Shimada H, Nakashizuka H, Hattori T, Mori R, Mizutani Y, Yuzawa M. Incidence of endophthalmitis after 20- and 25-gauge vitrectomy causes and prevention. Ophthalmology 2008; 115: 2215-2220.

11 Chan-Kai BT, Lauer AK. Transconjunctival, sutureless 25-gauge lens sparing vitrectomy for stage 4 retinopathy of prematurity-related retinal detachments. Retina 2009; 29: 854-859.

12 Iwahashi-Shima C, Miki A, Hamasaki T, Otori Y, Matsushita K, Kiuchi $\mathrm{Y}$ et al. Intraocular pressure elevation is a delayed-onset complication after successful vitrectomy for stages 4 and 5 retinopathy of prematurity. Retina 2012; 32: 1636-1642.

13 Azuma N, Ishikawa K, Hama Y, Hiraoka M, Suzuki Y, Nishina S. Early vitreous surgery for aggressive posterior retinopathy of prematurity. Am J Ophthalmol 2006; 142: 636-643.

14 Yokoi T, Yokoi T, Kobayashi Y, Nishina S, Azuma N. Risk factors for recurrent fibrovascular proliferation in aggressive posterior retinopathy of prematurity after early vitreous surgery. Am J Ophthalmol 2010; 150: 10-15.

15 Xu Y, Zhang Q, Kang X, Zhu Y, Li J, Chen Y et al. Early vitreoretinal surgery on vascularly active stage 4 retinopathy of prematurity through the preoperative intravitreal bevacizumab injection. Acta Ophthalmol 2013; 91: e304-e310.

16 Bhende P, Gopal L, Sharma T, Verma A, Biswas RK. Functional and anatomical outcomes after primary lens-sparing pars plana vitrectomy for Stage 4 retinopathy of prematurity. Indian J Ophthalmol 2009; 57: 267-271.

17 Kychenthal A, Dorta P. 25-gauge lens-sparing vitrectomy for stage 4 A retinopathy of prematurity. Retina 2008; 28: S65-S68.

18 El Rayes EN, Vinekar A, Capone A Jr. Three-year anatomic and visual outcomes after vitrectomy for stage 4B retinopathy of prematurity. Retina 2008; 28: 568-572.

19 Chow DR, Ferrone PJ, Trese MT. Refractive changes associated with scleral buckling and division in retinopathy of prematurity. Arch Ophthalmol 1998; 116: 1446-1448. 